\title{
Dealing Necessary and Sufficient Numbers of Cards for Sharing a One-Bit Secret Key (Extended Abstract)
}

\author{
Takaaki Mizuki $^{1}$, Hiroki Shizuya ${ }^{2}$, and Takao Nishizeki ${ }^{3}$ \\ 1 Nishizeki Lab., Graduate School of Information Sciences, Tohoku University, \\ Aoba-yama 05, Aoba-ku, Sendai 980-8579, Japan \\ mizuki@nishizeki.ecei.tohoku.ac.jp \\ 2 Education Center for Information Processing, Tohoku University, \\ Kawauchi, Aoba-ku, Sendai 980-8576, Japan \\ shizuya@ecip.tohoku.ac.jp \\ 3 Graduate School of Information Sciences, Tohoku University, \\ Aoba-yama 05, Aoba-ku, Sendai 980-8579, Japan \\ nishi@ecei.tohoku.ac.jp
}

\begin{abstract}
Using a random deal of cards to players and a computationally unlimited eavesdropper, all players wish to share a one-bit secret key which is information-theoretically secure from the eavesdropper. This can be done by a protocol to make several pairs of players share one-bit secret keys so that all these pairs form a spanning tree over players. In this paper we obtain a necessary and sufficient condition on the number of cards for the existence of such a protocol. Our condition immediately yields an efficient linear-time algorithm to determine whether there exists a protocol to achieve such a secret key sharing.
\end{abstract}

\section{Introduction}

Suppose that there are $k(\geq 2)$ players $P_{1}, P_{2}, \cdots, P_{k}$ and a passive eavesdropper, Eve, whose computational power is unlimited. All players wish to share a common one-bit secret key that is information-theoretically secure from Eve. Let $C$ be a set of $d$ distinct cards which are numbered from 1 to $d$. All cards in $C$ are randomly dealt to players $P_{1}, P_{2}, \cdots, P_{k}$ and Eve. We call a set of cards dealt to a player or Eve a hand. Let $C_{i} \subseteq C$ be $P_{i}$ 's hand, and let $C_{e} \subseteq C$ be Eve's hand. We denote this deal by $\mathcal{C}=\left(C_{1}, C_{2}, \cdots, C_{k} ; C_{e}\right)$. Clearly $\left\{C_{1}, C_{2}, \cdots, C_{k}, C_{e}\right\}$ is a partition of set $C$. We write $c_{i}=\left|C_{i}\right|$ for each $1 \leq i \leq k$ and $c_{e}=\left|C_{e}\right|$, where $|A|$ denotes the cardinality of a set $A$. Note that $c_{1}, c_{2}, \cdots, c_{k}$ and $c_{e}$ are the sizes of hands held by $P_{1}, P_{2}, \cdots, P_{k}$ and Eve respectively, and that $d=\sum_{i=1}^{k} c_{i}+c_{e}$. We call $\gamma=\left(c_{1}, c_{2}, \cdots, c_{k} ; c_{e}\right)$ the signature of deal $\mathcal{C}$. In this paper we assume that $c_{1} \geq c_{2} \geq \cdots \geq c_{k}$; if necessary, we rename the players. The set $C$ and the signature $\gamma$ are public to all the players and even to Eve, but the cards in the hand of a player or Eve are private to herself, as in the case of usual card games.

We consider a graph called a key exchange graph, in which each vertex $i$ represents a player $P_{i}$ and each edge $(i, j)$ joining vertices $i$ and $j$ represents a 


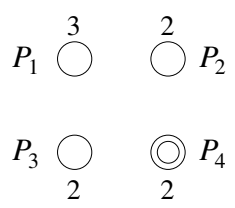

$\gamma=(3,2,2,2 ; 1)$

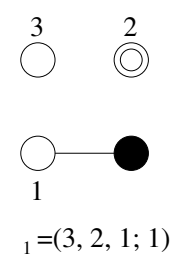

(b)

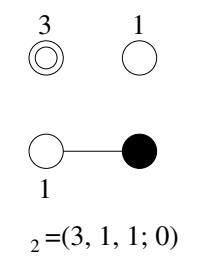

(c)

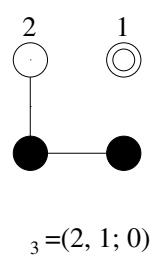

(d)

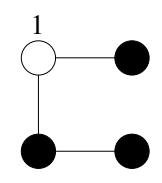

(e)

Fig. 1. A generating process of a key exchange graph.

pair of players $P_{i}$ and $P_{j}$ sharing a one-bit secret key $r_{i j} \in\{0,1\}$. (See Figure 1]) Refer to [8] for the graph-theoretic terminology. If the key exchange graph is a spanning tree as illustrated in Figure 1(e), then all the players can share a common one-bit secret key $r \in\{0,1\}$ as follows: an arbitrary player chooses a one-bit secret key $r \in\{0,1\}$, and sends it to the rest of the players along the spanning tree; when player $P_{i}$ sends $r$ to player $P_{j}$ along an edge $(i, j)$ of the spanning tree, $P_{i}$ computes the exclusive-or $r \oplus r_{i j}$ of $r$ and $r_{i j}$ and sends it to $P_{j}$, and $P_{j}$ obtains $r$ by computing $\left(r \oplus r_{i j}\right) \oplus r_{i j}$.

For the case $k=2$, Fischer, Paterson and Rackoff give a protocol to form a spanning tree, i.e. a graph having exactly one edge as the key exchange graph by using a random deal of cards [2].

Fischer and Wright [3]6] extend this protocol for any $k \geq 2$, and formalize a class of protocols called "key set protocols," a formal definition of which will be given in the succeeding section. Furthermore they give the so-called SFP protocol as a key set protocol. We say that a key set protocol works for a signature $\gamma$ if the protocol always forms a spanning tree as the key exchange graph for any deal $\mathcal{C}$ having the signature $\gamma[2 \sqrt[3]{4 / 5 \mid 6]}$. Let $\Gamma$ be a set of all signatures, where the number $k$ of players and the total number $d$ of dealt cards are taken over all values. Define sets $W$ and $L$ as follows:

$$
\begin{gathered}
W=\{\gamma \in \Gamma \mid \text { there is a key set protocol working for } \gamma\} ; \text { and } \\
L=\{\gamma \in \Gamma \mid \text { there is no key set protocol working for } \gamma\} .
\end{gathered}
$$

Thus $\{W, L\}$ is a partition of set $\Gamma$. Fischer and Wright show that their SFP protocol works for all $\gamma \in W$ [3,6]. Furthermore they prove that a sufficient condition for $\gamma \in W$ is $c_{k} \geq 1$ and $c_{1}+c_{k} \geq c_{e}+k$. They also show that it is a necessary and sufficient condition for the case $k=2$ [36]. However, a simple necessary and sufficient condition for the case $k \geq 3$ has not been known so far [3]6].

Since the SFP protocol works for all $\gamma \in W$, one can determine whether $\gamma \in W$ or not by simulating the SFP protocol for $\gamma$. However, it is necessary to simulate the protocol for all "malicious adversaries," and hence the time required by this simulation is exponential in $k$ and such a simulation is impractical.

In this paper for the case $k \geq 3$ we give a simple necessary and sufficient condition on a signature $\gamma$ for the existence of a key set protocol to work for $\gamma$. 
Given a signature $\gamma=\left(c_{1}, c_{2}, \cdots, c_{k} ; c_{e}\right)$, one can easily determine in time $O(k)$ whether $\gamma$ satisfies our condition or not. Thus our condition immediately yields an efficient linear-time algorithm for determining whether there exists a key set protocol to work for a given signature $\gamma$. Our condition looks in appearance to be similar to the condition for a given degree sequence to be "graphical," and the proof for our condition is complicated as well as those for a degree sequence 1.78 .

\section{Preliminaries}

In this section we explain the key set protocol formalized by Fischer and Wright, and present known results on this protocol [2,3,6].

We first define some terms. A key set $K=\{x, y\}$ consists of two cards $x$ and $y$, one in $C_{i}$, the other in $C_{j}$ with $i \neq j$, say $x \in C_{i}$ and $y \in C_{j}$. We say that a key set $K=\{x, y\}$ is opaque if $1 \leq i, j \leq k$ and Eve cannot determine whether $x \in C_{i}$ or $x \in C_{j}$ with probability greater than $1 / 2$. Note that both players $P_{i}$ and $P_{j}$ know that $x \in C_{i}$ and $y \in C_{j}$. If $K$ is an opaque key set, then $P_{i}$ and $P_{j}$ can share a one-bit secret key $r_{i j} \in\{0,1\}$, using the following rule agreed on before starting the protocol: $r_{i j}=0$ if $x>y ; r_{i j}=1$, otherwise. Since Eve cannot determine whether $r_{i j}=0$ or $r_{i j}=1$ with probability greater than $1 / 2$, the secret key $r_{i j}$ is information-theoretically secure. We say that a card $x$ is discarded if all the players agree that $x$ has been removed from someone's hand, that is, $x \notin\left(\cup_{i=1}^{k} C_{i}\right) \cup C_{e}$. We say that a player $P_{i}$ drops out of the protocol if she no longer participates in the protocol. We denote by $V$ the set of indices $i$ of all the players $P_{i}$ remaining in the protocol. Note that $V=\{1,2, \cdots, k\}$ before starting a protocol.

The key set protocol has four steps as follows.

1. Choose a player $P_{s}, s \in V$, as a proposer by a certain procedure.

2. The proposer $P_{s}$ determines in mind two cards $x, y$. The cards are randomly picked so that $x$ is in her hand and $y$ is not in her hand, i.e. $x \in C_{s}$ and $y \in\left(\bigcup_{i \in V-\{s\}} C_{i}\right) \cup C_{e}$. Then $P_{s}$ proposes $K=\{x, y\}$ as a key set to all the players. (The key set is proposed just as a set. Actually it is sorted in some order, for example in ascending order, so Eve learns nothing about which card belongs to $C_{s}$ unless Eve holds $y$.)

3. If there exists a player $P_{t}$ holding $y$, then $P_{t}$ accepts $K$. Since $K$ is an opaque key set, $P_{s}$ and $P_{t}$ can share a one-bit secret key $r_{s t}$ that is informationtheoretically secure from Eve. (In this case an edge $(s, t)$ is added to the key exchange graph.) Both cards $x$ and $y$ are discarded. Let $P_{i}$ be either $P_{s}$ or $P_{t}$ that holds a smaller hand; if $P_{s}$ and $P_{t}$ hold hands of the same size, let $P_{i}$ be the proposer $P_{s} . P_{i}$ discards all her cards and drops out of the protocol. Set $V:=V-\{i\}$. Return to step 1 .

4. If there exists no player holding $y$, that is, Eve holds $y$, then both cards $x$ and $y$ are discarded. Return to step 1. (In this case no new edge is added to the key exchange graph.) 
These steps 1-4 are repeated until either exactly one player remains in the protocol or there are not enough cards left to complete step 2 even if two or more players remain. In the first case the key exchange graph becomes a spanning tree. In the second case the protocol fails to form a spanning tree.

We now illustrate the execution of the key set protocol. Let $\gamma=(3,2,2,2 ; 1)$ be the signature before starting the protocol. Thus there are four players $P_{1}, P_{2}$, $P_{3}, P_{4}$ and Eve; $P_{1}$ has a hand of size $3, P_{2}, P_{3}$ and $P_{4}$ have hands of size 2 , and Eve has a hand of size 1 . At the beginning of the protocol the key exchange graph has four isolated vertices and has no edge, as illustrated in Figure 1(a). In Figure 1] a white circle represents a vertex corresponding to a player remaining in the protocol, and the number attached to a white circle represents the size of the corresponding player's hand. Suppose that $P_{4}$ is chosen as a proposer in step 1. In Figure 1 a double white circle represents the vertex corresponding to a proposer. In step $2, P_{4}$ proposes $K=\{x, y\}$ such that $x \in C_{4}$ and $y \notin C_{4}$. Assume that $y \in C_{3}$. Then step 3 is executed, $P_{3}$ and $P_{4}$ share a one-bit secret key $r_{34}$, and edge $(3,4)$ is added to the key exchange graph, as illustrated in Figure 1(b). Since both cards $x$ and $y$ are discarded, the sizes of hands of both $P_{3}$ and $P_{4}$ decrease by one. Further, since the size of $P_{3}$ 's hand was the same as that of $P_{4}$ 's hand, the proposer $P_{4}$ discards all her cards and drops out of the protocol. Thus the resulting signature is $\gamma_{1}=(3,2,1 ; 1)$. In Figure 1 a black circle represents a vertex corresponding to a player who has dropped out of the protocol. We now return to step 1. Assume that $P_{2}$ is chosen as a proposer and $y \in C_{e}$. Then step 4 is executed, and the sizes of hands of both $P_{2}$ and Eve decrease by one. Thus the resulting signature is $\gamma_{2}=(3,1,1 ; 0)$, and no new edge is added to the key exchange graph, as illustrated in Figure 1 (c). Since step 4 terminates, we now return to step 1 . Assume that $P_{1}$ is chosen as a proposer and $y \in C_{3}$. Then edge $(1,3)$ is added to the key exchange graph as illustrated in Figure 1(d). Since the size of $P_{1}$ 's hand decreases by one and $P_{3}$ drops out of the protocol, the resulting signature is $\gamma_{3}=(2,1 ; 0)$. We now return to step 1. Assume that $P_{2}$ is chosen as a proposer. Then $y \in C_{1}$ because only $P_{1}$ and $P_{2}$ remain in the protocol and Eve's hand has already been empty. Thus edge $(1,2)$ is added to the key exchange graph, and the key exchange graph becomes a spanning tree, as illustrated in Figure 1(e). Thus the protocol terminates. As seen from the example above, during the execution of the key set protocol, each connected component of the key exchange graph always has exactly one vertex (drawn in a white circle) corresponding to a player remaining in the protocol.

Considering various procedures for choosing the proposer $P_{s}$ in step 1, we obtain the class of key set protocols.

First consider the procedure in step 1 for the case $k=2$. Fischer, Paterson and Rackoff show that, if the procedure always chooses the player with the larger hand as a proposer $P_{s}$, then the resulting key set protocol works for any signature $\gamma=\left(c_{1}, c_{2} ; c_{e}\right)$ such that $c_{2} \geq 1$ and $c_{1}+c_{2} \geq c_{e}+2$ [2]. On the other hand, one can easily see that if there exists a key set protocol working for a signature $\gamma=\left(c_{1}, c_{2} ; c_{e}\right)$ then $c_{2} \geq 1$ and $c_{1}+c_{2} \geq c_{e}+2$. Thus the following Theorem 1 holds [3]. 
Theorem 1. [3] Let $k=2$. Then $\gamma \in W$ if and only if $c_{2} \geq 1$ and $c_{1}+c_{2} \geq c_{e}+2$.

Next consider the procedure in step 1 for the case $k \geq 3$. As a key set protocol, Fischer and Wright give the SFP (smallest feasible player) procedure which chooses the "feasible" player with the smallest hand as a proposer $P_{s}[3,6]$. Let $\gamma=\left(c_{1}, c_{2}, \cdots, c_{k} ; c_{e}\right)$ be the current signature. If $c_{e} \geq 1, P_{i}$ with $c_{i}=1$ were chosen as a proposer, and $y \in C_{e}$ occurred, then $P_{i}$ 's hand would become empty although she remains in the protocol, and hence the key exchange graph would not become a spanning tree. On the other hand, if $c_{e}=0$, then $y \in C_{e}$ does not occur and hence the procedure appears to be able to choose $P_{i}$ with $c_{i}=1$ as a proposer; however, if $y \in C_{j}$ and $c_{j}=1$, then $P_{j}$ 's hand would become empty and hence the key exchange graph would not become a spanning tree. Thus the procedure can choose $P_{i}$ with $c_{i}=1$ as a proposer only when $c_{e}=0$ and $c_{j} \geq 2$ for every $j$ such that $1 \leq j \leq k$ and $j \neq i$, that is, only when $i=k$ and $c_{k-1} \geq 2$. Remember that $c_{1} \geq c_{2} \geq \cdots \geq c_{k}$ is assumed. Hence, we say that player $P_{i}$ is feasible if the following condition (1) or (2) holds.

(1) $c_{i} \geq 2$.

(2) $c_{e}=0, c_{i}=1$ with $i=k$, and $c_{k-1} \geq 2$.

Thus, if the hands of all the players remaining in the protocol are not empty, i.e. $c_{k} \geq 1$, and the proposer $P_{s}$ is feasible, then the hands of all the players remaining in the protocol will not be empty at the beginning of the succeeding execution of steps $1-4$.

We define a mapping $f$ from $\Gamma$ to natural numbers, as follows: $f(\gamma)=i$ if $P_{i}$ is the feasible player with the smallest hand (ties are broken by selecting the player having the largest index); and $f(\gamma)=0$ if there is no feasible player. For example, if $\gamma=(4,3,2,2,1,1 ; 3)$, then $f(\gamma)=4$. If $\gamma=(4,4,3,3,1 ; 0)$, then $f(\gamma)=k=5$ because $c_{e}=0, c_{k}=1$ and $c_{k-1} \geq 2$. If $\gamma=(1,1,1 ; 2)$, then $f(\gamma)=0$ because there is no feasible player. Hereafter we often denote $f(\gamma)$ simply by $f$.

From now on let $\gamma=\left(c_{1}, c_{2}, \cdots, c_{k} ; c_{e}\right)$. Note that the definition of $f$ immediately implies the following Lemma 2 Lemma 2(a) provides a trivial necessary condition for $\gamma \in W$.

Lemma 2. The following (a) and (b) hold.

(a) If $k \geq 3$ and $\gamma \in W$, then $c_{k} \geq 1$ and $f(\gamma) \geq 1$ [3].

(b) If $c_{k} \geq 1$, then $c_{i}=1$ for every $i$ such that $f(\gamma)+1 \leq i \leq k$.

The SFP procedure chooses a proposer $P_{s}$ as follows:

$$
s= \begin{cases}f(\gamma) & \text { if } 1 \leq f(\gamma) \leq k \\ 1 & \text { if } f(\gamma)=0\end{cases}
$$

The key set protocol resulting from this procedure is called the SFP protocol. The following Theorem 3 has been known on the SFP protocol [36]. 
Theorem 3. [36] Let $\gamma \in \Gamma$. Then there exists a key set protocol working for $\gamma$, i.e. $\gamma \in W$, if and only if the SFP protocol works for $\gamma$.

Furthermore the following Lemma 4 is known on a sufficient condition for $\gamma \in W[36$.

Lemma 4. 36] If $c_{k} \geq 1$ and $c_{1}+c_{k} \geq c_{e}+k$, then $\gamma \in W$.

The sufficient condition in Lemma 4 is not a necessary condition in general. For example, $\gamma=(3,3,2,1 ; 1)$ does not satisfy the condition in Lemma 4 but the SFP protocol works for $\gamma$ and hence $\gamma \in W[36$. In this paper we obtain a simple necessary and sufficient condition for $\gamma \in W$ for any $k \geq 3$. As shown later, $\gamma=(3,3,2,1 ; 1)$ satisfies our necessary and sufficient condition.

\section{$3 \quad$ Necessary and Sufficient Condition}

For $k=3$, we obtain the following Theorem 5 on a necessary and sufficient condition for $\gamma \in W$.

Theorem 5. Let $k=3$. Then $\gamma \in W$ if and only if $c_{3} \geq 1$ and $c_{1}+c_{3} \geq c_{e}+3$.

Proof. Given in Section 5.

For $k \geq 4$, we obtain the following Theorem 6 on a necessary and sufficient condition for $\gamma \in W$. Hereafter let $B=\left\{i \in V \mid c_{i}=2\right\}$, and let $b=\lfloor|B| / 2\rfloor$. Note that, by Lemma 2 (a), a trivial necessary condition for $\gamma \in W$ is $c_{k} \geq 1$ and $f(\gamma) \geq 1$.

Theorem 6. Let $k \geq 4, c_{k} \geq 1$ and $f \geq 1$. Then $\gamma \in W$ if and only if

$$
\sum_{i=1}^{\tilde{f}} \max \left\{c_{i}-h^{+}, 0\right\} \geq \tilde{f},
$$

where

$$
\begin{gathered}
\bar{f}=f-\delta, \\
\tilde{f}=\bar{f}-2 \epsilon, \\
h=c_{e}-c_{k}+k-\bar{f}, \\
h^{+}=h+\epsilon, \\
\delta=\left\{\begin{array}{l}
0 \text { if } f=1 ; \\
1 \text { if } 2 \leq f \leq k-1 ; \\
2 \text { if } f=k \text { and } c_{k-1} \geq c_{k}+1 ; \text { and } \\
3 \text { if } f=k \text { and } c_{k-1}=c_{k},
\end{array}\right.
\end{gathered}
$$




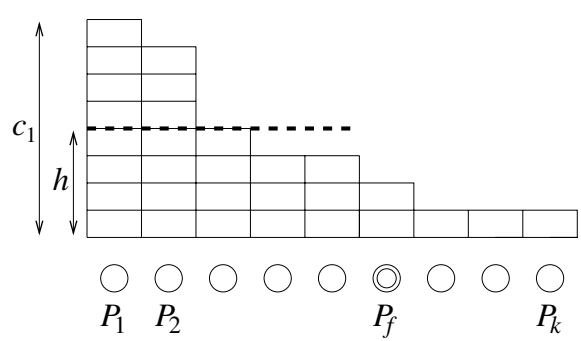

(a)

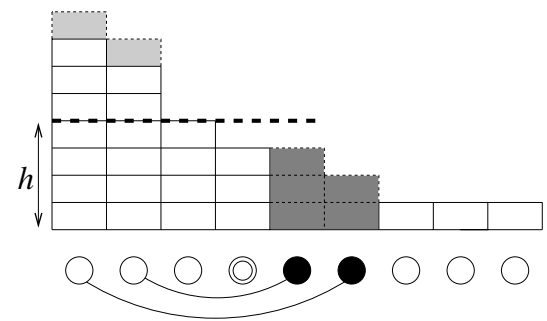

(c)

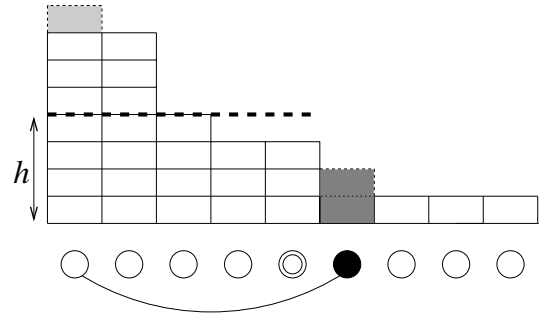

(b)

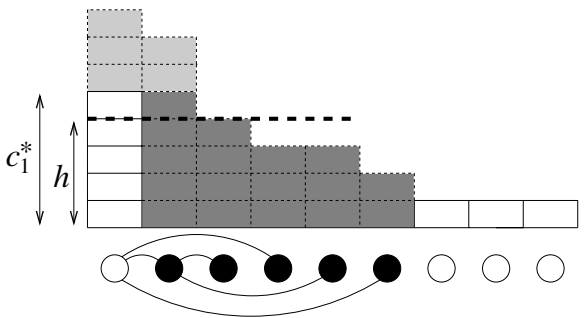

(d)

Fig. 2. The evolution of a key exchange graph and the alteration of a signature.

and

$$
\epsilon= \begin{cases}\max \left\{\min \left\{c_{2}-h, b\right\}, 0\right\} & \text { if } 5 \leq f \leq k-1 \\ \max \left\{\min \left\{c_{2}-h, b-1\right\}, 0\right\} & \text { if } 5 \leq f=k \text { and } c_{e} \geq 1 ; \text { and } \\ 0 & \text { otherwise. }\end{cases}
$$

Proof. Given in Section 6.

[Remark] Since $c_{1} \geq c_{2} \geq \cdots \geq c_{k}$ is assumed, Eq. (11) is equivalent to

$$
\sum_{i=1}^{k} \max \left\{c_{i}-h^{+}, 0\right\} \geq \widetilde{f}
$$

where the summation is taken over all $i, 1 \leq i \leq k$, although the summation in Eq. (1) is taken over all $i, 1 \leq i \leq \widetilde{f}$.

Figure 2 (a) illustrates Eq. (1); the left hand side of Eq. (11) is equal to the number of cards above the dotted line in Figure2(a) where the rectangles stacked on a player $P_{i}, 1 \leq i \leq k$, represent the cards of $P_{i}$ 's hand.

As mentioned in Section 2, the SFP protocol works for $\gamma=(3,3,2,1 ; 1)$, but $\gamma$ does not satisfy the sufficient condition in Lemma 4 [3.6]. By the definition of $f$ we have $f=f(\gamma)=3$. Since $k=4$, we have $2 \leq f=3=k-1$, and hence by Eq. (6) $\delta=1$. By Eq. (2) $\bar{f}=3-1=2$, and by Eq. (4) $h=1-1+4-2=2$. Since $f=3<5$, by Eq. (7) we have $\epsilon=0$. Hence by Eq. (3) we have $\tilde{f}=2-0=2$ and 
by Eq. (5) $h^{+}=2+0=2$. Therefore $\sum_{i=1}^{\tilde{f}} \max \left\{c_{i}-h^{+}, 0\right\}=(3-2)+(3-2)=$ $2=\widetilde{f}$. Thus $\gamma$ satisfies Eq. (1), the necessary and sufficient condition in Theorem 6

The following Corollary 7 follows from Theorems 1 , 5 and 6. This corollary provides a necessary and sufficient condition for $\gamma \in W$ under a natural assumption that all players have hands of the same size.

Corollary 7. Let $k \geq 2$ and $c_{1}=c_{2}=\cdots=c_{k}$. Then $\gamma \in W$ if and only if

$$
c_{1} \geq\left\{\begin{array}{l}
c_{e} / 2+1 \quad \text { if } k=2 ; \\
c_{e} / 2+3 / 2 \text { if } k=3 ; \text { and } \\
c_{e} / 2+2 \quad \text { if } k \geq 4 .
\end{array}\right.
$$

Corollary 7 means that the required size $c_{1}$ of hands is the same for any $k \geq 4$ when $c_{1}=c_{2}=\cdots=c_{k}$. Note that the total number $k c_{1}$ of required cards increases when $k$ increases.

The following Corollary 8 is immediate from Corollary 7

Corollary 8. Let $k \geq 2$ and $c_{1}=c_{2}=\cdots=c_{k}=c_{e}$. Then $\gamma \in W$ if and only if

$$
c_{1} \geq\left\{\begin{array}{l}
2 \text { if } k=2 ; \\
3 \text { if } k=3 ; \text { and } \\
4 \text { if } k \geq 4 .
\end{array}\right.
$$

\section{Malicious Adversary}

In this paper we use a malicious adversary in order to prove Theorem 6 .

If a key set protocol works for a signature $\gamma$, then the key exchange graph must become a spanning tree for any deal $\mathcal{C}$ having the signature $\gamma$. Hence, whoever has the card $y$ contained in the proposed key set $K=\{x, y\}$, the key exchange graph should become a spanning tree. The malicious adversary determines who holds the card $y$. Considering a malicious adversary to make it hard for the key exchange graph to become a spanning tree, we obtain a necessary condition for $\gamma \in W$. On the other hand, if under some condition on a signature $\gamma$ a key set protocol always forms a spanning tree as the key exchange graph for any malicious adversary, then the condition is a sufficient one for $\gamma \in W$.

We use a function $\mathcal{A}: \Gamma \times V \rightarrow V \cup\{e\}$ to represent a malicious adversary, as follows. Remember that $\Gamma$ is the set of all signatures and that $V$ is the set of indices of all the players remaining in a protocol. Let $e$ be Eve's index. The inputs to the function $\mathcal{A}(\gamma, s)$ are the current signature $\gamma \in \Gamma$ and the index $s \in V$ of a proposer $P_{s}$ chosen in the protocol. Its output is either the index $t$ of a player $P_{t}$ remaining in the protocol or the index $e$ of Eve; $\mathcal{A}(\gamma, s)=t \neq e$ means that player $P_{t}$ holds card $y$; and $\mathcal{A}(\gamma, s)=e$ means that Eve holds card $y$.

From now on, we denote by $\gamma=\left(c_{1}, c_{2}, \cdots, c_{k} ; c_{e}\right)$ the current signature, and denote by $\gamma_{(s, \mathcal{A})}^{\prime}=\left(c_{1}^{\prime}, c_{2}^{\prime}, \cdots, c_{k^{\prime}}^{\prime} ; c_{e}^{\prime}\right)$ the resulting signature after executing 
steps 1-4 under the assumption that $P_{s}$ proposes a key set $K=\{x, y\}$ and $y \in C_{\mathcal{A}(\gamma, s)}$.

The definition of a malicious adversary $\mathcal{A}$ immediately implies the following Lemma 9 .

Lemma 9. Let $k \geq 3$. Then $\gamma \in W$ if and only if there exists a proposer $P_{s}$ such that $\gamma_{(s, \mathcal{A})}^{\prime} \in W$ for any malicious adversary $\mathcal{A}$. That is,

$$
\gamma \in W \Longleftrightarrow \exists s \forall \mathcal{A} \quad \gamma_{(s, \mathcal{A})}^{\prime} \in W
$$

in other words,

$$
\gamma \in L \Longleftrightarrow \forall s \exists \mathcal{A} \quad \gamma_{(s, \mathcal{A})}^{\prime} \in L .
$$

From now on let $k \geq 3$. If $f=0$, then by Lemma 2 (a) $\gamma \in L$. On the other hand, if $f \geq 1$, then the index $s$ of the proposer $P_{s}$ chosen by the SFP procedure satisfies $s=f$. Furthermore, by Theorem 3 the SFP protocol works for all $\gamma \in W$. Thus, if $\gamma \in W$, then $\gamma_{(f, \mathcal{A})}^{\prime} \in W$ for any malicious adversary $\mathcal{A}$. Hence, the following Corollary 10 immediately follows from Theorem 3 .

Corollary 10. Let $k \geq 3$ and $f(\gamma) \geq 1$. Then $\gamma \in W$ if and only if $\gamma_{(f, \mathcal{A})}^{\prime} \in W$ for any malicious adversary $\mathcal{A}$. That is,

$$
\gamma \in W \Longleftrightarrow \forall \mathcal{A} \quad \gamma_{(f, \mathcal{A})}^{\prime} \in W
$$

in other words,

$$
\gamma \in L \Longleftrightarrow \exists \mathcal{A} \quad \gamma_{(f, \mathcal{A})}^{\prime} \in L
$$

It follows from the definition of a key set protocol that if two players $P_{i}$ and $P_{j}$ hold hands of the same size, that is, $c_{i}=c_{j}$, then

$$
\forall \mathcal{A} \quad \gamma_{(i, \mathcal{A})}^{\prime} \in W \Longleftrightarrow \forall \mathcal{A} \quad \gamma_{(j, \mathcal{A})}^{\prime} \in W .
$$

Hence, if there exist two or more players $P_{i}$ with $c_{i}=c_{s}$ (including the proposer $P_{s}$ ), then one may assume without loss of generality that $P_{s}$ has the largest index among all these players. We call it Assumption 1 for convenience sake. Furthermore, if $\mathcal{A}(\gamma, s)=t \neq e$ and there exist two or more players $P_{i}$ with $c_{i}=c_{t}$ and $i \neq s$ (including $P_{t}$ ), then one may assume without loss of generality that $P_{t}$ has the largest index among all these players. We call it Assumption 2 for convenience sake. Under the two assumptions above, $\gamma_{(s, \mathcal{A})}^{\prime}=\left(c_{1}^{\prime}, c_{2}^{\prime}, \cdots, c_{k^{\prime}}^{\prime} ; c_{e}^{\prime}\right)$ satisfies $c_{1}^{\prime} \geq c_{2}^{\prime} \geq \cdots \geq c_{k^{\prime}}^{\prime}$ since $\gamma$ satisfies $c_{1} \geq c_{2} \geq \cdots \geq c_{k}$.

The total size $\sum_{i=1}^{k} c_{i}$ of all the players' hands decreases by two or more if $\mathcal{A}(\gamma, s)=t \neq e$; it decreases by exactly one if $\mathcal{A}(\gamma, s)=e$. If a key set protocol works for $\gamma$, then $\mathcal{A}(\gamma, s)=t \neq e$ occurs $k-1$ times until the protocol terminates because the key exchange graph becomes a spanning tree having $k-1$ edges at the end of the protocol. Furthermore $\mathcal{A}(\gamma, s)=e$ would occur $c_{e}$ times. Hence, if a key set protocol works for $\gamma$, then $\sum_{i=1}^{k} c_{i} \geq 2(k-1)+c_{e}=c_{e}+2 k-2$. Thus we have the following Lemma 11 as a trivial necessary condition for $\gamma \in W$.

Lemma 11. If $\gamma \in W$, then $\sum_{i=1}^{k} c_{i} \geq c_{e}+2 k-2$. 


\section{Proof of Theorem 5}

In this section we give a proof of Theorem [5]

Since Lemma 4 implies the sufficiency of the condition in Theorem 5 , we prove its necessity. That is, we show that if $k=3$ and $\gamma \in W$ then $c_{3} \geq 1$ and $c_{1}+c_{3} \geq c_{e}+3$. In order to prove this, we use the following malicious adversary $\mathcal{A}^{*}$ :

$$
\mathcal{A}^{*}(\gamma, s)=\left\{\begin{array}{l}
3 \text { if } s=1 \\
1 \text { if } s=2 \\
e \text { if } s=3
\end{array}\right.
$$

We first have the following Lemma 12.

Lemma 12. Let $k=3, c_{3} \geq 1$ and $c_{1}+c_{3} \leq c_{e}+2$. Then the following (a) or (b) holds.

(a) $\gamma \in L$.

(b) $\gamma_{\left(f, \mathcal{A}^{*}\right)}^{\prime}$ satisfies $k^{\prime}=3, c_{3}^{\prime} \geq 1$ and $c_{1}^{\prime}+c_{3}^{\prime} \leq c_{e}^{\prime}+2$.

Proof. Let $k=3, c_{3} \geq 1$ and $c_{1}+c_{3} \leq c_{e}+2$. If $f=0$, then $\gamma \in L$ by Lemma 2 (a). Thus one may assume that $1 \leq f \leq 3$. Then there are the following three cases.

Case 1: $f=1$.

In this case, by Lemma 2(b), we have $\gamma=\left(c_{1}, 1,1 ; c_{e}\right)$ and hence $c_{2}=c_{3}=1$. Thus, by $c_{1}+c_{3} \leq c_{e}+2$ we have $c_{1} \leq c_{e}+1$. Hence $\sum_{i=1}^{3} c_{i} \leq\left(c_{e}+1\right)+1+1=$ $c_{e}+2 k-3$. Therefore $\gamma \in L$ by Lemma 11

Case 2: $f=2$.

In this case, by Lemma 2(b) we have $\gamma=\left(c_{1}, c_{2}, 1 ; c_{e}\right)$. Since $f=2$, the definition of $f$ implies $c_{2} \geq 2$ and $c_{e} \geq 1$. Furthermore, since $c_{3}=1$ and $c_{1}+c_{3} \leq c_{e}+2$, we have $c_{1} \leq c_{e}+1$. Since $f=2$, let $P_{2}$ be a proposer $P_{s}$. Since $\mathcal{A}^{*}(\gamma, s)=1$ for $s=2$, the size of the hand of $P_{1}$ holding card $y$ decreases by one and the proposer $P_{2}$ drops out of the protocol, and hence $\gamma_{\left(f, \mathcal{A}^{*}\right)}^{\prime}=\left(c_{1}-1,1 ; c_{e}\right)$. Therefore $c_{1}^{\prime}+c_{2}^{\prime}=\left(c_{1}-1\right)+1=c_{1}$. Since $c_{1} \leq c_{e}+1$ and $c_{e}^{\prime}=c_{e}$, we have $c_{1}^{\prime}+c_{2}^{\prime} \leq c_{e}^{\prime}+1$. Thus by Theorem $1 \gamma_{\left(f, \mathcal{A}^{*}\right)}^{\prime} \in L$. Therefore Corollary 10 implies $\gamma \in L$.

Case 3: $f=3$.

In this case $c_{e} \geq 1$; if $c_{e}=0$, then by $c_{3} \geq 1$ and $c_{1}+c_{3} \leq c_{e}+2=2$ we have $c_{1}=c_{2}=c_{3}=1$, and hence $f=0$, contrary to $f=3$. Since $f=3$, let $P_{3}$ be a proposer. Since $\mathcal{A}^{*}(\gamma, s)=e$ for $s=3$, the sizes of the hands of both $P_{3}$ and Eve decrease by one, and hence $\gamma_{\left(f, \mathcal{A}^{*}\right)}^{\prime}=\left(c_{1}, c_{2}, c_{3}-1 ; c_{e}-1\right), k^{\prime}=3$ and $c_{e}^{\prime}=c_{e}-1$. Since $P_{3}$ was feasible, we have $c_{3}^{\prime}=c_{3}-1 \geq 1$. Furthermore $c_{1}^{\prime}+c_{3}^{\prime}=c_{1}+\left(c_{3}-1\right) \leq\left(c_{e}+2\right)-1=c_{e}^{\prime}+2$. Thus (b) holds.

Define the size $\operatorname{size}(\gamma)$ of a signature $\gamma$ as follows: $\operatorname{size}(\gamma)=c_{e}+k$.

We are now ready to prove the necessity of the condition in Theorem 5 . 


\section{(Proof for the necessity of the condition in Theorem 5 )}

Let $k=3$. We shall show that if $c_{3}=0$ or $c_{1}+c_{3} \leq c_{e}+2$ then $\gamma \in L$. If $c_{3}=0$, then Lemma 2 (a) implies $\gamma \in L$. Therefore it suffices to prove the following claim: if $c_{3} \geq 1$ and $c_{1}+c_{3} \leq c_{e}+2$ then $\gamma \in L$. We prove the claim by induction on $\operatorname{size}(\gamma)=c_{e}+k$. Let $c_{3} \geq 1$ and $c_{1}+c_{3} \leq c_{e}+2$. Since $k=3$, $\operatorname{size}(\gamma) \geq 3$.

First consider the case $\operatorname{size}(\gamma)=3$. In this case, $c_{e}=0$, and hence $c_{1}+c_{3} \leq$ $c_{e}+2=2$. Thus $c_{1}=c_{2}=c_{3}=1$, and hence $f=0$. Therefore by Lemma 2(a) $\gamma \in L$. $l-1$.

Next let $l \geq 4$, and assume inductively that the claim holds when $\operatorname{size}(\gamma)=$

Consider any signature $\gamma \operatorname{such}$ that $\operatorname{size}(\gamma)=l$. By Lemma 12 the following (a) or (b) holds:

(a) $\gamma \in L$; and

(b) $\gamma_{\left(f, \mathcal{A}^{*}\right)}^{\prime}$ satisfies $k^{\prime}=3, c_{3}^{\prime} \geq 1$ and $c_{1}^{\prime}+c_{3}^{\prime} \leq c_{e}^{\prime}+2$.

Thus one may assume that (b) holds. Then, $\operatorname{since} \operatorname{size}\left(\gamma^{\prime}\right)=\operatorname{size}(\gamma)-1=l-1$, by the induction hypothesis we have $\gamma_{\left(f, \mathcal{A}^{*}\right)}^{\prime} \in L$. Therefore Corollary 10 implies $\gamma \in L$.

\section{Sketchy Proof of Theorem 6}

In this section we outline a proof of Theorem 6

One can easily prove Theorem 6 for the case $f=1$ as follows. Let $k \geq 4$, $c_{k} \geq 1$ and $f=1$. Then $\delta=\epsilon=0$ and hence $\widetilde{f}=\bar{f}=f=1$. By Lemma 2(b) $c_{k}=1$ and hence $h^{+}=h=c_{e}-1+k-1=c_{e}+k-2$. Thus, Eq. (11) is equivalent to $\max \left\{c_{1}-c_{e}-k+2,0\right\} \geq 1$, and hence equivalent to $c_{1} \geq c_{e}+k-1$. Therefore Theorem 6 for the case $f=1$ immediately follows from the following Lemma 13.

Lemma 13. Let $c_{k} \geq 1$ and $f=1$. Then $\gamma \in W$ if and only if $c_{1} \geq c_{e}+k-1$.

Proof. The sufficiency immediately follows from Lemma 4 . Therefore it suffices to prove the necessity. Let $c_{k} \geq 1, f=1$ and $\gamma \in W$. Then by Lemma 11] we have $\sum_{i=1}^{k} c_{i} \geq c_{e}+2 k-2$. On the other hand, since $f=1$, by Lemma 2 (b) $\gamma=\left(c_{1}, 1,1, \cdots, 1 ; c_{e}\right)$ and hence $\sum_{i=1}^{k} c_{i}=c_{1}+k-1$. Therefore, $c_{1}+k-1 \geq$ $c_{e}+2 k-2$ and hence $c_{1} \geq c_{e}+k-1$.

We then sketch a proof of Theorem 6 for the case $2 \leq f \leq k$. The detail is omitted in this extended abstract. We sketch a proof only for the necessity of the condition in Theorem 6. (One can prove the sufficiency by induction on $\operatorname{size}(\gamma)=c_{e}+k$.) Let $k \geq 4, c_{k} \geq 1,2 \leq f \leq k$ and $\gamma \in W$. Instead of proving Eq. (1) we prove the following equation holds:

$$
\sum_{i=1}^{\bar{f}} \max \left\{c_{i}-h, 0\right\} \geq \bar{f},
$$


which is obtained from Eq. (II) by replacing $\tilde{f}$ and $h^{+}$with $\bar{f}$ and $h$, respectively.

For simplicity, we assume that $\delta=1$, i.e. $2 \leq f \leq k-1$. (The proof for $\delta=2,3$ is similar.) Then by Lemma 2(b) $c_{k}=1$. Furthermore $\bar{f}=f-1$ and $h=c_{e}+k-f$. Thus Eq. (9) is equivalent to

$$
\sum_{i=1}^{f-1} \max \left\{c_{i}-\left(c_{e}+k-f\right), 0\right\} \geq f-1 .
$$

We prove the necessity of Eq. (10). Let $2 \leq f \leq k-1$. Then the signature is $\gamma=\left(c_{1}, c_{2}, \cdots, c_{f}, 1,1, \cdots, 1 ; c_{e}\right)$. That is, there are exactly $f$ feasible players $P_{1}, P_{2}, \cdots, P_{f}$, and each of the remaining $k-f$ players $P_{f+1}, P_{f+2}, \cdots, P_{k}$ has exactly one card. The key exchange graph has exactly $k$ isolated vertices before starting the protocol, as illustrated in Figure2(a). In Figure 2, a white rectangle represents a card in players' hands. The SFP protocol chooses the feasible player $P_{f}$ with the smallest hand as a proposer. Consider a malicious adversary that does not choose Eve and always chooses the player with the largest hand as $P_{t}$ with $y \in C_{t}$. Then $P_{f}$ and the player $P_{t}$ with the largest hand share a one-bit secret key, the size of $P_{t}$ 's hand decreases by one, $P_{f}$ drops out of the protocol, and an edge joining two vertices corresponding to these two players is added to the key exchange graph, as illustrated in Figure 2(b). In the example of Figure 2, the size of $P_{1}$ 's hand decreases by one, $P_{f}$ discards all her cards and drops out of the protocol, and edge $(1, f)$ is added to the key exchange graph. In Figure 2(b), we lightly shade the rectangle corresponding to the card $y$ discarded by $P_{t}=P_{1}$, and darkly shade the rectangles corresponding to the cards discarded by $P_{f}$ who drops out of the protocol. At the next execution of steps $1-4$, the proposer is $P_{f-1}$. By considering the same malicious adversary as above, $P_{f-1}$ and the player with the largest hand share a one-bit secret key as illustrated in Figure 2(c). In Figure 2 (b), since $P_{1}$ has a hand of the same size as $P_{2}$, by Assumption $2 P_{t}=P_{2}$ and hence edge $(2, f-1)$ is added to the key exchange graph as illustrated in Figure 2 (c). Repeat such an operation until $P_{1}$ becomes a proposer, i.e. there exists exactly one feasible player as illustrated in Figure 2(d), and let $\gamma^{*}=\left(c_{1}^{*}, c_{2}^{*}, \cdots, c_{k^{*}}^{*} ; c_{e}\right)$ be the resulting signature. Then $k^{*}=k-f+1, c_{2}^{*}=c_{3}^{*}=\cdots=c_{k^{*}}^{*}=1, f\left(\gamma^{*}\right)=1$, and the size of Eve's hand remains $c_{e}$. By Corollary 10 we have $\gamma^{*} \in W$. Therefore, by Lemma 13 , $c_{1}^{*} \geq c_{e}+k^{*}-1=c_{e}+k-f=h$. The malicious adversary has chosen $f-1$ players $P_{i}$ in total as $P_{t}$ so far, and hence there are exactly $f-1$ lightly shaded rectangles in Figure 2(d). The malicious adversary above implies that such a player $P_{i}, 1 \leq i \leq f-1$, should have a hand of size greater than $h$ when she was chosen by the malicious adversary. Thus there are $f-1$ or more rectangles above the dotted line in Figure 2(a). Therefore we have $\sum_{i=1}^{f-1} \max \left\{c_{i}-h, 0\right\} \geq f-1$, and hence Eq. (10) holds.

We have sketched a proof of the necessity of Eq. (9). One can similarly prove the necessity of Eq. (1). 


\section{Conclusion}

In this paper we gave a simple necessary and sufficient condition on signature $\gamma=\left(c_{1}, c_{2}, \cdots, c_{k} ; c_{e}\right)$ for the existence of a key set protocol to work for $\gamma$. In other words we gave a simple complete characterization of the sets $W$ and $L$.

Since the SFP protocol works for all $\gamma \in W$ (Theorem 3), one can determine whether $\gamma \in W$ or not by simulating the SFP protocol for $\gamma$. However, it is necessary to simulate the protocol for all malicious adversaries, and hence the time required by this simulation is exponential in $k$ and such a simulation is impractical. Clearly one can determine in time $O(k)$ whether our necessary and sufficient condition, i.e. Eq. (1) or (8), holds or not. Thus one can determine in time $O(k)$ whether $\gamma \in W$ or not.

This paper addresses only the class of key set protocols, and hence it still remains open to obtain a necessary and sufficient condition for any (not necessarily key set) protocol to work for $\gamma[5]$.

An Eulerian circuit is more appropriate as a key exchange graph than a spanning tree if it is necessary to acknowledge the secure key distribution. We have given a protocol to achieve such a key exchange [9].

\section{References}

1. T. Asano, "An $O(n \log \log n)$ time algorithm for constructing a graph of maximum connectivity with prescribed degrees," J. Comput. and Syst. Sci., 51, pp. 503-510, 1995.

2. M. J. Fischer, M. S. Paterson and C. Rackoff, "Secret bit transmission using a random deal of cards," DIMACS Series in Discrete Mathematics and Theoretical Computer Science, AMS, 2, pp. 173-181, 1991.

3. M. J. Fischer and R. N. Wright, "An application of game-theoretic techniques to cryptography," DIMACS Series in Discrete Mathematics and Theoretical Computer Science, AMS, 13, pp. 99-118, 1993.

4. M. J. Fischer and R. N. Wright, "An efficient protocol for unconditionally secure secret key exchange," Proceedings of the 4th Annual Symposium on Discrete Algorithms, pp. 475-483, 1993.

5. M. J. Fischer and R. N. Wright, "Bounds on secret key exchange using a random deal of cards," J. Cryptology, 9, pp. 71-99, 1996.

6. M. J. Fischer and R. N. Wright, "Multiparty secret key exchange using a random deal of cards," Proc. Crypto '91, Lecture Notes in Computer Science, SpringerVerlag, 576, pp. 141-155, 1992.

7. S. L. Hakimi, "On realizability of a set of integers as degrees of the vertices of a linear graph. I," J. SIAM Appl. Math., 10, 3, pp. 496-506, 1962.

8. F. Harary, "Graph Theory," Addison-Wesley, Reading, Mass., 1969.

9. T. Mizuki, H. Shizuya and T. Nishizeki, "Eulerian secret key exchange," Proc. COCOON '98, Lecture Notes in Computer Science, Springer, 1449, pp. 349-360, 1998.

10. E. F. Schmeichel and S. L. Hakimi, "On planar graphical degree sequences," SIAM J. Appl. Math., 32, 3, pp. 598-609, 1977. 